\title{
INTERNET OF THINGS ARCHITECTURE BASED CLOUD FOR HEALTHCARE
}

\author{
Istabraq M. Al-Joboury ${ }^{1}$, Emad H. Al-Hemiary ${ }^{2}$ \\ 1,2 Department of Networks Engineering, Al-Nahrain University, Iraq \\ estabriq_94@coie-nahrain.edu.iq ${ }^{1}$, emad@coie-nahrain.edu.iq ${ }^{2}$ \\ (Received: 16/01/2018; Accepted: 12/03/2018)
}

\begin{abstract}
The Internet of Things (IoT) contains smart devices placed in different environments, connected with each other across networks and Internet. The integration between Things and Cloud Computing (CC) for monitoring and permanent storage is required for future IoT applications. Therefore, this paper proposes IoT architecture based Cloud for healthcare network when patients are remotely monitored by their family and physicians. This proposed architecture is different from the traditional IoT architecture that consists of Things, getaways, middleware, and application layers which in turn need connectivity insurance between them. The proposed architecture is designed and configured using Cisco Packet Tracer version 7.0 over two sites: Site 'A' located at smart home and site 'B' located at the smart hospital. The results show that the IoT based Cloud enhances the patient life style by using smart sensors and mobile application, as well as the physicians can remotely monitor the data in real time.
\end{abstract}

Keywords- Internet of Things; Cloud Computing; IoT Architecture; Embedded Devices; Packet Tracer; HTTP; Monitoring.

\section{INTRODUCTION}

The Internet of Things (IoT) connects all the physical devices such as sensors, actuators, microcontrollers (or socalled Things) to the Internet, therefore the IoT becomes the source of Information from these Things. The Radio Frequency Identification (RFID) was the first created IoT concept to unique identify other Things. Then, the Global Positioning System (GPS) was created to identify the location of humans or Things. However, in the meantime the IoT Things have been growing rapidly and these Things includes all domains of life such as healthcare, e-learning, industrial, etc. [1]. The medical Things generates data of the patients to be recorded, managed, monitored, and remotely controlled in smart hospitals. In addition, physicians and patient's family can observe the status condition of the patient. Therefore, the Wireless Body Area Network (WBAN) would be a part of IoT concept. The medical sensors can be placed on the patient's body and connected to Internet using WiFi, Bluetooth, ZigBee, etc. [2].

The IoT concept optimized the healthcare by providing real time monitoring to data from sensors [4]. IoT architecture has three main layers, namely: Perception layer, consists of two sublayers. The first sublayer is about data collecting from the medical Things, Patients, nurses, hospital, and so on. Then, these data from sensors are transmit to the gateways [5]. The network layer includes all routing and switching devices and protocols to forward data from the perception layer to another network so that for example, patient's family located at different area of hospital could receive the data of their patient [6]. Finally, application layer which represents the management, monitoring, and decision-making depends on the data from the perception layer in order to notify the patient [7].

The studies refer that the world will have around 7 trillion IoT Things by 2020 and expected that each user will have 1000 Things [8]. Therefore, Cloud Computing (CC) becomes a critical need to provide a numerous storage and facility data sharing [9]. The innovation is to integrate the CC and IoT. It is expected to optimize the healthcare system by reducing the power, cost, and complexity [10]. CC can be centralized and located at different area or country of smart hospital. $\mathrm{CC}$ has three different types of services, namely: Software as a Service (SaaS), in this type all 
networking, computing, and storage, are controlled by the third party such as Gmail. Platform as a Services (PaaS), in this type only the data and application controlled by the user such as Amazon Cloud. Finally, (Infrastructure as a Service-IaaS), in this type the storage is controlled by the third party, and all other services are controlled by the user [11].

It can be seen that the IoT layers are different from the Cloud layer and therefore it is essential to provide assurance connectivity [12]. We propose IoT architecture based on Cloud for storage, control, and monitoring the patient. The proposed architecture is designed and configured using Cisco Packet Tracer version 7.0 [13] over three sites: Site A located at smart home, site B located at smart hospital, while site C located at different area of smart hospital. The Cisco Packet Tracer is chosen because it includes the IoT, Cloud, and OSI layers, as well as supports several programming languages of sensors such as python, Java Script, and visual Scripting. Other IoT simulators like IoT Simulator [14], and SimpleIoTSimulator [15] which include only the Things and Cloud layers can be used as alternatives to Cisco packet tracer.

The rest of this paper is organized as follows: Section II covers related works. Section III, proposes IoT architecture based on Cloud. Section IV, shows the results of this proposed architecture. Section VI, discusses the proposed architecture. Finally, section VII concludes this paper.

\section{LITERATURE REVIEW}

This section presents the literature review related to this paper. There are a few papers uses the Cisco packet tracer to simulate the IoT application. In [16], the authors verify the proposed architecture for asthma patients based Cloud using Cisco packet tracer. In this proposed architecture, temperature sensor is used to sense the temperature values from the patient and sent to Cloud through wireless router. In addition, the authors implement this proposed architecture practically. However, the architecture does not consider multiple sensors running simultaneously, as well as monitoring the system in real time in Cisco packet tracer. In [17] the authors use IoT concept in educational filed. That paper proposes architecture with Raspberry Pi microcontroller instead of using the traditional Personal Computers (PCs) in order to reduce cost. This work is implemented using Cisco packet tracer, as well as practically. Finally, in [18] the authors introduce the Cisco packet tracer simulation tool to simulate the IoT environment with the help of Microcontrollers (MCUs) such as Raspberry pi and Arduino with sensors and actuators.

\section{IOT BASED CLOUd ARCHITECTURE}

The integration between Things and Cloud for monitoring and permanent storage is required for future IoT applications. Therefore, IoT based Cloud architecture is presented for healthcare network when patients are remotely monitored by their family and physicians. This proposed architecture is different from the traditional IoT architecture which requires connectivity insurance between layers. The traditional IoT has five layers: Things, gateway, middleware, application, and business layers. These layers have limited capabilities in storage and energy. Things are a low cost and light-weight devices which can store few bytes of messages locally.

The proposed architecture uses several different medical Things integrated with each other to provide a valuable information and connected to the Internet through network gateway. To enhance the traditional IoT in terms of 
processing and storage, this proposed architecture integrates Cloud layer with traditional IoT architecture. Cloud can provide real time and realistic services when integrated with IoT. In this proposed architecture, Cloud layer provides a permanent storage and makes messages accessible everywhere over the Internet. CC has different layers than IoT, thus connectivity between IoT and Cloud layers are required by using API, frameworks, protocols, or software. Several tools are provided to monitor normal and abnormal messages in real time globally from Cloud or locally from Things. This proposed architecture not only provides storage and monitoring, but also humans can contact with each other using smart devices or emails.

The proposed architecture is configured and run using Cisco Packet Tracer version 7.0 over two sites: Site 'A' located at smart home, and site 'B' located at smart hospital. The layers of proposed architecture are as follows (Fig. 1):

\section{A. Things Layer}

This layer consists of sensors that are placed on patient's body, and in smart home located at site 'A' to provide continuous messaging. The transmitted messages can be monitored using smart devices such as phones and tablets and stored in Cloud servers. This layer consists of three medical and two home sensors (sensors called IoE in the Cisco Packet Tracer). The three medical sensors, namely: Patient's Implanted Glucose Meter, fitness (which includes respiration rate and exercise level), and temperature sensors are used to collect messages from the patient's body. In addition, the two home sensors, namely: Electronic door and video camera provide patient monitoring. These sensors are programmed using Java Script language in Cisco Packet Tracer, and the main functions used to program the sensors are as shown in Table I. The IP addressing for sensors is obtained through DHCP service configured inside APs which takes the network identity $192.168 .0 .0 / 24$.

TABLE I

MAIN FUNCTIONS USED IN THINGS LAYER

\begin{tabular}{|c|c|}
\hline Function & Meaning \\
\hline Get & $\begin{array}{l}\text { Gets messages with units of sensors from the simulation Cisco Packet Tracer, for instance the } \\
\text { messages of glucose meter sensor maps between ( } 40 \text { to } 500) \text { in Milligrams per Deciliter (mg/dl) }\end{array}$ \\
\hline API & $\begin{array}{l}\text { Connects the Things layer to the Cloud layer so that Cloud layer receives the messages and } \\
\text { provides a remote control }\end{array}$ \\
\hline Callback & Processes the response from Cloud layer to Things layer \\
\hline States & $\begin{array}{l}\text { Reports the states of Things layer to the Cloud layer, for instance sends the states of electronic } \\
\text { door sensor: close, open, locked or unlocked. In addition, if the sensor does not work properly, it } \\
\text { sends the problem to Cloud layer }\end{array}$ \\
\hline
\end{tabular}

\section{B. Gateway Layer}

For smart monitoring, the Things layer needs Internet access so that the physicians and patients` family can receive messages from sensors. Things layer is connected to two APs, namely: Digital Life Controllers (two DLC-100) located at site 'A'. DLC-100 is a modern device and supports automation packages. AP transfers these messages from Things layer using WiFi (IEEE802.11n) technology to Cloud layer. Cisco switch (2960-24TT) is included in this proposed architecture to connect both APs to modem PT by Fast Ethernet cable. Then, the modem PT is connected to the 
Internet. The IP addressing for getaways layer is obtained through DHCP service configuration from hospital router which takes the network identity 209.165.200.224/27.

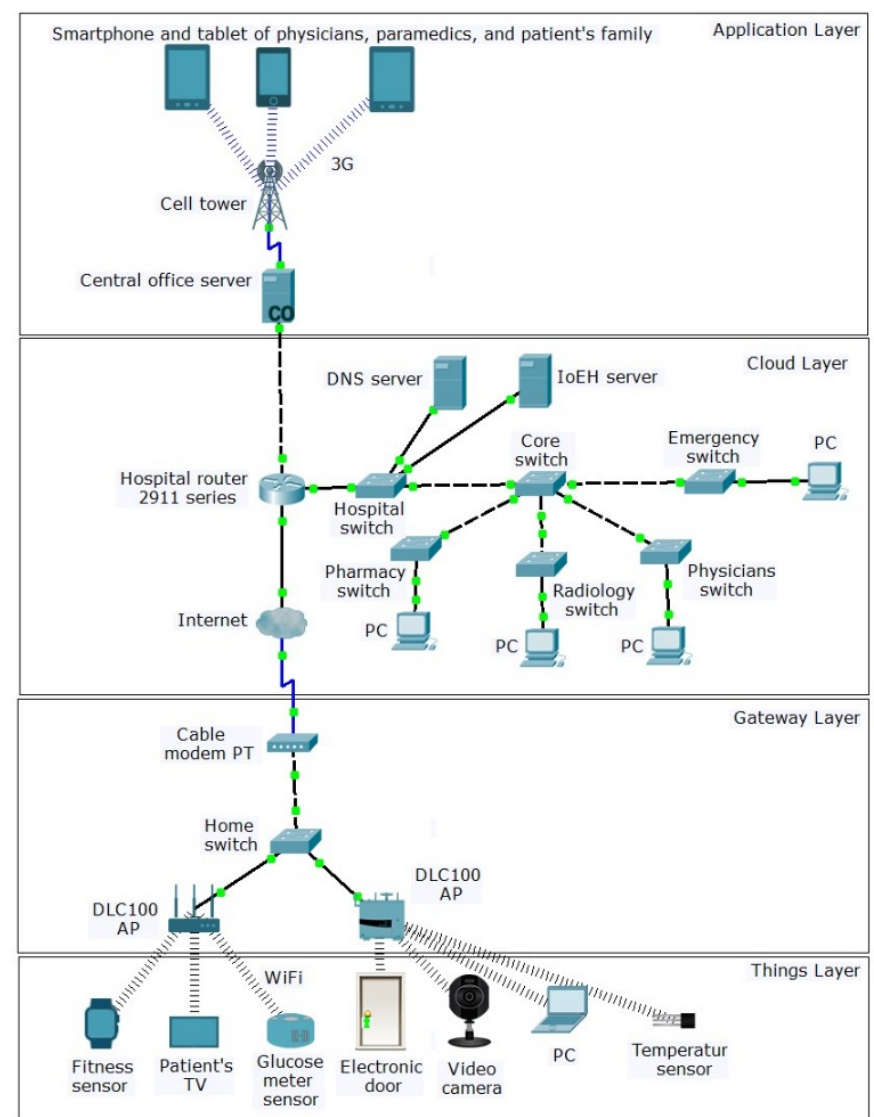

Figure 1. Simulated IoT healthcare network showing the integration of Cloud layer with traditional IoT architecture

\section{Cloud Layer}

This layer connects two sites ('A', and 'B') together through the router 2911 series (named in the architecture hospital router). DHCP is configured in the hospital router to distribute IP addresses from the pools to the connected networks. CELL and WAN pools are assigned to the cell tower layer and the gateway layer respectively. From the other end, the hospital router is connected to a switch (named in architecture hospital switch) located at site 'B'. Then, hospital switch is connected to another switch (named in architecture core switch) and followed by four switches for four medical departments which are: physicians, emergency room, pharmacy, and radiology. The type of all switches of this proposed architecture is Cisco catalyst 2960-24TT.

This layer consists of two servers (PaaS) located at site 'B'. The IoEH server has three services, namely IoE, email, and HTTP. Where, IoE feature is used to remote control Things layer so that sensors can be controlled by physicians as shown in Fig. 2. Each sensor can register itself on IoEH server using username and password authentication. As an example, the electronic lock of the door can be remotely opened or closed when the paramedics arrived at urgent need 
(this type is called M2M interaction). The smart hospital allows the patient to take the right decisions by sending the right instructions based on messages coming from sensors (this type is called Machine to Human (M2H)). However, patient may regularly visit or contact his physician to ensure that the treatment fits his condition (this type called Human to Human $(\mathrm{H} 2 \mathrm{H})$ ). Finally, all messages from the sensors are stored in Cloud servers. The HTTP protocol is used for communication and to browse messages.

The email service is used for exchanging mail messages between physicians and patient. The email service uses SMTP for sending and processing outgoing mails and uses POP3 for receiving and handling incoming mails. Thus, each patient has an email identity in the hospital database. The simulation includes another service named DNS which is used to map domain names into IP, for example "coie-nahrain.edu.iq" is mapped to 5.10.230.122. The network identity of this layer is $10.0 .0 .0 / 24$.

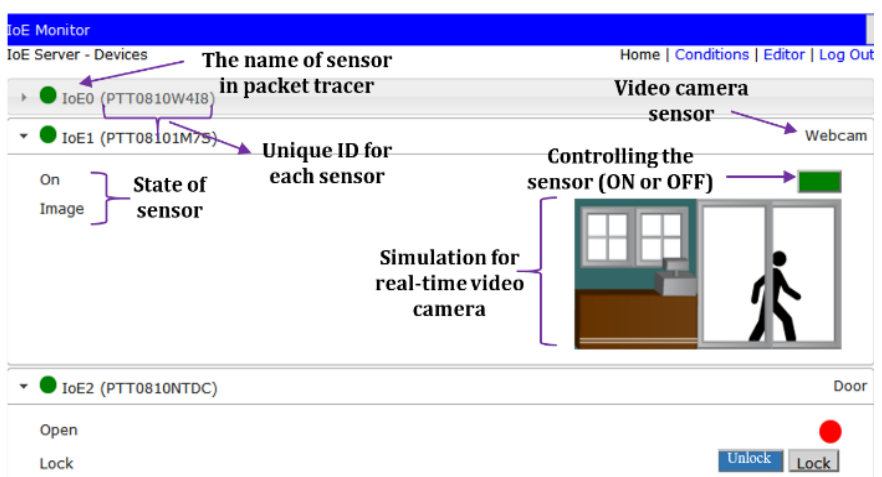

Figure 2. Smart monitoring and control of sensors from Things layer using Packet Tracer.

\section{Application Layer}

The smart hospital is connected to the central office server and cell tower for making local and long distance calls using the Third Generation (3G). The IP addressing for cell tower layer is obtained through DHCP service configuration from hospital router which takes the network identity 209.165.201.224/27.

The patient's family and physician can monitor messages from sensors using smart devices. However, if there is an abnormal condition, notification is sent to the smart devices. In addition, an ambulance is sent to the patient's home when the patient's condition does not improve. The video camera sensor assists the paramedics to find the patient's location in site 'A'. The monitoring tools are installed and configured on phones and tablets using Cisco Packet Tracer as shown in the next section. Finally, Cisco Packet Tracer can show messages from sensors through graphs.

Flow of packets in the proposed IoT based Cloud architecture is discussed in steps below:

1) Things are connected to the nearer AP in home, then packets from these things are sent to AP that Things connected to it.

2) Smart devices like mobile receive messages from Things to monitoring packets locally at home.

3) Packets from AP are reached to switch in home, and its turn sends packets to modem PT to enable Internet.

4) Hospital router receives packets from Things layer through modem PT, then forwards these packets to several medical departments. In addition, two servers in hospital receive packets to provide different services like email. 
5) Also, Hospital router forwards packets from Things to central office server to enable smart monitoring through Internet and $3 \mathrm{G}$.

\section{Results}

The results of the IoT based Cloud architecture show for healthcare network using mobile application installed on smartphone and configured to monitor the patient status. First of all, Ping program is used to test reachability between different layers in the simulated IoT network. Fig. 3 shows an example where the command line of Ping is used from the PC at home to test connectivity to the IoEH server.

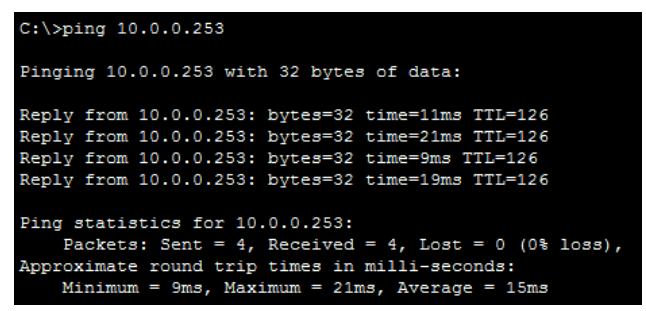

Figure 3. Pinging connectivity between PC at home to IoEH server located at Cloud layer

Messages from three sensors (glucose meter, respiratory in Breaths per Minute (BPM), and exercise levels) are shown in Fig. 4. For clarification, there are three levels of the exercise sensor (low, medium, and high). These messages can be monitored by the patient himself, patient's family, and physicians in real time at home, hospital, or anywhere using smartphone or tablet. It can be seen from Fig. 4 that all messages of sensors are within the normal levels for the patient status. If the messages are out of the normal ranges, a warning message appears in this mobile application to notify the recipient in order to take action immediately as shown in Fig. 5. Fig. 6 shows sensors reading averaged per five hours to help the intended recipient in making decisions. The five-hour period can be adjusted in the simulation environment and can be set according to the patient's condition. The respiratory rate and exercise level can be monitored in the patient's smart watch so that the patient can observe his levels in real time from anywhere. Fig. 7 (a) presents the messages within normal range. The smart watch is connected to the glucose sensor. If the sugar level gets very high or very low, a warning message is sent to the intended recipients as shown in Fig. 7 (b). Furthermore, if the patient status is not getting well, the paramedics are sent to the patient location.

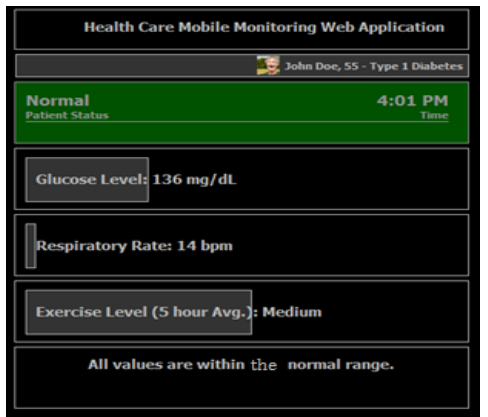

Figure 4. Mobile application in smart phones and tablets using Packet Tracer

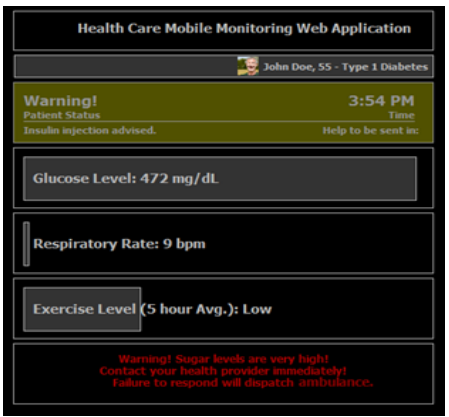

Figure 5. Warring message appearing in mobile to monitor abnormal messages using Packet Tracer 
Finally, the simulation can demonstrate sensor readings per day. This result is programmed in Packet Tracer to enable record archiving and per day statistics. The physician can look at the per day readings to decide if the patient needs more attention. Fig. 8 shows a sample result of respiration sensor where the $\mathrm{x}$-axis is an hour time scale and the $\mathrm{y}$-axis is the breathing rate in BPM. The simulation assumes that this rate changes during the day to highlight graph significance.so that the patient can observe his levels in real time from anywhere. Fig. 7 (a) presents the messages within normal range. The smart watch is connected to the glucose sensor. If the sugar level gets very high or very low, a warning message is sent to the intended recipients as shown in Fig. 7 (b). Furthermore, if the patient status is not getting well, the paramedics are sent to the patient location.

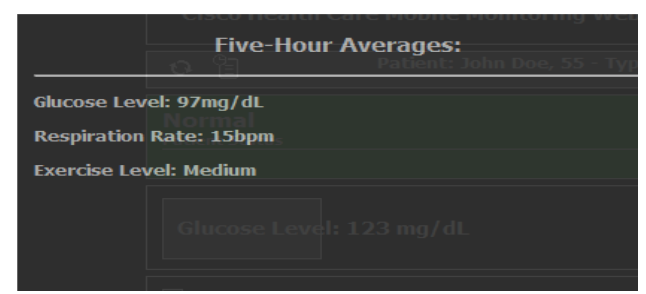

Figure 6. Mobile application provides the average messages of sensors per five hours using Packet Tracer

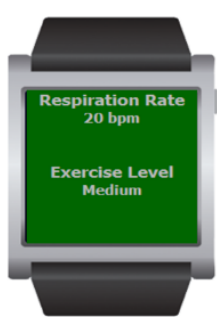

(a)

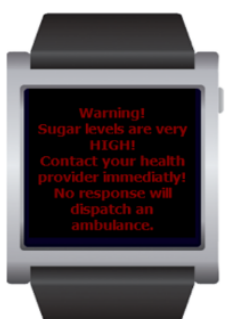

(b)

Figure 7. Patient's smartwatch for monitoring sensors using Packet Tracer:

(a) Messages of sensors within normal range (b) A warning message appearing for out of normal range messages

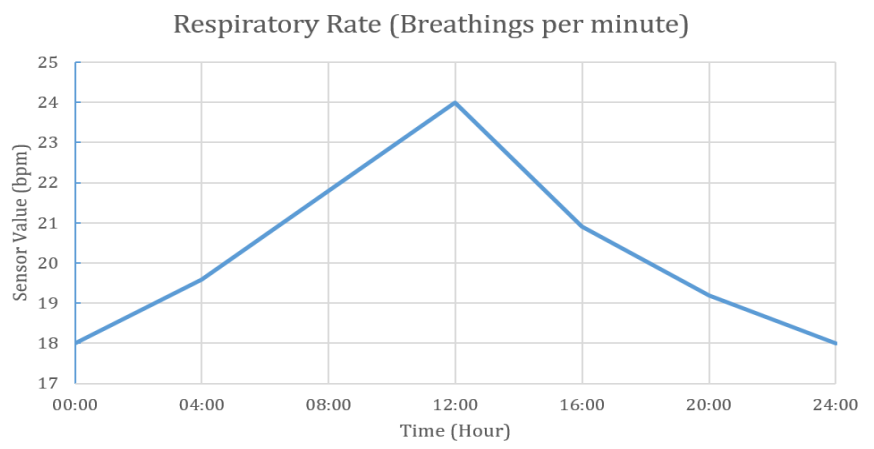

Figure 8. History of values of respiratory sensor using Packet Tracer

\section{DISCUSSION}

IoT Based Cloud architecture for the healthcare network is simulated using Cisco Packet Tracer. Although the simulation is intended for healthcare networks, it can be adopted for any field such as transportation, smart home, etc. by changing the type of the sensor in the Things layer. Cisco Packet Tracer simulates the IoT technology and provides 
smart monitoring without the complexity of hardware. The simulation provides a dashboard environment to control messages from sensor such as a glucose meter sensor. This dashboard provides multiple situations like the induce Hypoglycemia, induce Hyperglycemia, or normal levels as shown in Fig. 9. The architecture is tested under different situations and prove validity of simulation with IoT based Cloud architecture. The previous works implemented IoT architecture based on two layers: Things and gateway and remote control was not included, while the contribution of this paper is to design and implement IoT architecture based on four layers: Things, gateway, Cloud, and application as well as remote control, notification, monitoring, and decisions-making are included. In addition, programming functions in this proposed to automate IoT sensors and several protocols to configure networking layers in order to connect to Internet and Cloud with its services, in contrast the previous works are just to monitor data from Things layer locally.

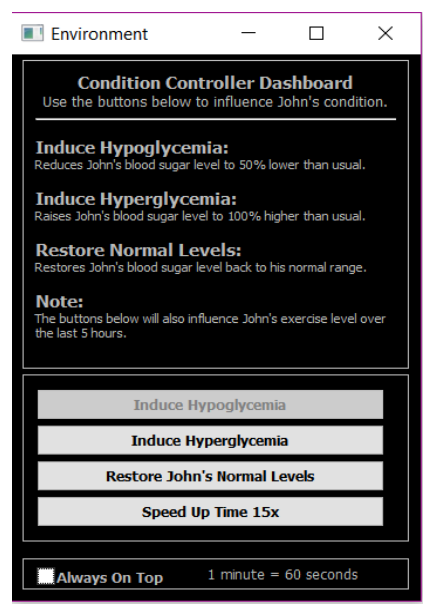

Figure 9. Condition controller dashboard of messages on sensors using Packet Tracer

\section{Conclusion}

This paper proposes an IoT architecture for healthcare network over three different sites, namely: Smart home, smart hospital, and different area of smart hospital. The data from Things are needed to be stored for processing and monitoring the patients. The result show that integration between the IoT and Cloud enhances the real-time monitoring. The sensors that are placed on patient's body or at smart home can be remotely monitored. The proposed IoT architecture based Cloud is designed and simulated using Cisco Packet Tracer. The assurance connectivity between IoT and Cloud layers is provided. Monitoring tools, mobile and smartwatch application are provided in order to observe the values of sensors. Notify is sent to the patient and the paramedics when there is an abnormal value.

\section{REFERENCES}

[1] J. Qi, P. Yang, G. Min, O. Amft, F. Dong, and L. Xu, “Advanced internet of things for personalised healthcare system: A survey," Pervasive and Mobile Computing, 2017

[2] S. F. Khan, "Health care monitoring system in Internet of Things (IoT) by using RFID," 2017 6th International Conference on Industrial Technology and Management (ICITM), 2017.

[3] B. Tan, A. Burrows, R. Piechocki, I. Craddock, Q. Chen, K. Woodbridge, and K. Chetty, "Wi-Fi based passive human motion sensing for inhome healthcare applications," 2015 IEEE 2nd World Forum on Internet of Things (WF-IoT), 2015.

[4] I. M. Al-Joboury and E. H. Al-Hemiary, "Internet of Things (IoT): Readme," Qalaai Zanist Scientific Journal, vol. 2, no. 2, pp. 343-358, 2017.

[5] A. Al-Fuqaha, M. Guizani, M. Mohammadi, M. Aledhari and M. Ayyash, "Internet of Things: A Survey on Enabling Technologies, Protocols, and Applications", IEEE Communications Surveys \& Tutorials, vol. 17, no. 4, pp. 2347-2376, 2015. 
[6] M. Aazam, I. Khan, A. A. Alsaffar, and E.-N. Huh, "Cloud of Things: Integrating Internet of Things and cloud computing and the issues involved," Proceedings of 2014 11th International Bhurban Conference on Applied Sciences \& Technology (IBCAST) Islamabad, Pakistan, 14th - 18th January, 2014.

[7] J. Kaur, and K. Kaur, "Internet of Things: A Review on Technologies, Architecture, Challenges, Applications, FutureTrends," International journal of Computer Network and Information Security, pp. 57-70, 2017.

[8] H. Sun, X. Wang, R. Buyya, and J. Su, "CloudEyes: Cloud-based malware detection with reversible sketch for resource-constrained internet of things (IoT) devices," Software: Practice and Experience, vol. 47, no. 3, pp. 421-441, 2016.

[9] Y. Yu, "Mobile edge computing towards 5G: Vision, recent progress, and open challenges," China Communications, vol. 13, no. 2, pp. 8999, 2016.

[10] J. H. Abawajy and M. M. Hassan, "Federated Internet of Things and Cloud Computing Pervasive Patient Health Monitoring System," IEEE Communications Magazine, vol. 55, no. 1, pp. 48-53, 2017.

[11] C. Stergiou and K. E. Psannis, "Recent advances delivered by Mobile Cloud Computing and Internet of Things for Big Data applications: a survey," International Journal of Network Management, vol. 27, no. 3, May 2016.

[12] I. M. Al-Joboury and E. H. Al-Hemiary, "F2CDM: Internet of Things for Healthcare Network Based Fog-to-Cloud and Data-in-Motion Using MQTT Protocol," Ubiquitous Networking Lecture Notes in Computer Science, pp. 368-379, 2017.

[13] Cisco packet tracer, last visited: 2017/8/1, http://www.packettracernetwork.com/features/packettracer-7-newfeatures.html.

[14] MIMIC IoT Simulator, last visited: 2017/8/1, http://www.gambitcomm.com/site/iot simulator.php.

[15] SimpleIoTSimulator, last visited: 2017/8/1, http://www.smplsft.com/SimpleIoTSimulator.html.

[16] T. G. AL-Jaf and E. H. Al-Hemiary, "Internet of Things Based Cloud Smart Monitoring for Asthma Patient," Qalaai Zanist Scientific Journal, vol. 2, no. 2, pp. 359 - 364, 2017.

[17] A. Ali, and S. S. Hassan, "IoT Based Smart Projectors," International Journal of Scientific \& Engineering Research, volume 8, Issue 6, 2017.

[18] T. R. Babu, Laxmiganesh, and A. Gowrisanka, "Iot for Self Monitoring Analysis Remote Transducers," International Journal of Engineering and Technical Research (IJETR), P.P 2321-0869, vol. 6, no. 3, 2016. 